\title{
Use of scanning laser ophthalmoscopy to monitor papilloedema in idiopathic intracranial hypertension
}

\author{
D A Mulholland, J J Craig, S J A Rankin
}

\begin{abstract}
Aims-To determine the sensitivity of confocal scanning laser ophthalmoscopy (SLO) in detecting clinically significant changes in papilloedema secondary to idiopathic intracranial hypertension (IIH) and the correlation with visual field loss. Methods-Eight patients-three new, two recurrent, and three chronic cases of IIH-were examined over a 9 month period with SLO (Heidelberg retina tomograph) of optic nerve head and 30-2 Humphrey visual fields (six cases). Optic disc swelling (volume) was assessed in each eye using a circular contour line placed around the swollen optic nerve head on the mean image of three topographic images. Nine volume measurements from single images in each eye of every patient were performed on one occasion to assess repeatability.
\end{abstract}

Results-In the five acute cases optic disc volumes (range 1-16 $\mathrm{mm}^{3}$ ) decreased with treatment to stable, normal levels. Three of these had mild, reproducible, field defects which resolved. Two chronic cases had stable or fluctuating disc volume with no detectable change in grade of papilloedema and mild field loss. In one case which underwent theco-peritoneal shunting both disc volume and field worsened, indicating therapeutic failure. Both improved postoperatively.

Conclusions-SLO has a high sensitivity for detecting small changes in disc volumes and correlates closely with visual field change in the short term. It can confirm therapeutic failure by detecting stable or increasing disc volume. Decreasing volume may indicate resolution of papilloedema or secondary optic atrophy, so accompanying funduscopy and visual fields remain essential.

(Br f Ophthalmol 1998;82:1301-1305)

Idiopathic intracranial hypertension (IIH) is a condition characterised by raised intracranial pressure with its associated symptoms and signs in the absence of an intracranial mass or hydrocephalus and normal cerebrospinal fluid. Visual loss secondary to papilloedema and subsequent optic atrophy is the main complication and the incidence of severe, irreversible, bilateral visual loss has been reported from $4 \%{ }^{1}$ to $10 \% .^{2}$ In most patients automated perimetric techniques detect less severe defects which are often reversible. ${ }^{1}$ Visual moni- toring by Goldmann or automated perimetry and optic disc examination is mandatory in all patients, since we cannot reliably predict those patients who will develop significant visual loss. ${ }^{134}$ Several factors have been noted to be associated with visual loss-recent weight gain, ${ }^{1}$ race and sex, black males, ${ }^{5}$ severe papilloedema, and optic atrophy. ${ }^{6} 7$

Degree of optic disc swelling has not consistently been related to visual loss ${ }^{18}$ but to date no method of quantifying disc swelling has been available. Clinical grading of papilloedema using the methods of Sanders ${ }^{9}$ or Frisén ${ }^{10}$ is not sensitive enough to describe very small, short term changes. Quantification of optic disc swelling may give an early indication of worsening or response to treatment in IIH. Confocal scanning laser ophthalmoscopy (SLO) is a new technique for analysing retinal and optic disc topography. We have studied the use of SLO in this condition to assess usefulness as a monitoring method.

\section{Patients and methods}

We studied eight patients with IIH. All fulfilled the modified Dandy Criteria for diagnosis. ${ }^{11}$ Three were new cases (patients 1, 2, and 3), two recurrent (patients 5 and 6 ), and three cases had been diagnosed at least 6 months previously and had chronic disc swelling despite treatment (patients 4, 7, and 8). Six patients were followed with Humphrey 30-2 automated perimetry and in two patients who had difficulty with this method Goldmann perimetry was performed. The clinical features are outlined in Table 1.

The same individual (DM) performed all the testing. Baseline assessment consisted of full ophthalmological examination including best refracted Snellen visual acuity, stereoscopic optic disc assessment and stereo disc photography, perimetry and topographic imaging of the optic nerve head (SLO). Follow up assessments were performed at variable intervals ( 2 weeks -3 months) depending on clinical stability. At each examination visual acuity, slit lamp biomicroscopy of optic disc, perimetry, and SLO were repeated.

Confocal SLO using the Heidelberg retina tomograph (Heidelberg Engineering) was employed. This technique and reproducibility have been described. ${ }^{12}{ }^{13}$ This involves a scanning diode laser which obtains a topographic image of the optic nerve head consisting of 32 aligned, transverse, optical sections over a depth of $1-4 \mathrm{~mm}$ depending on the height of swelling. The required field was 10,
Accepted for publication 2 April 1998 

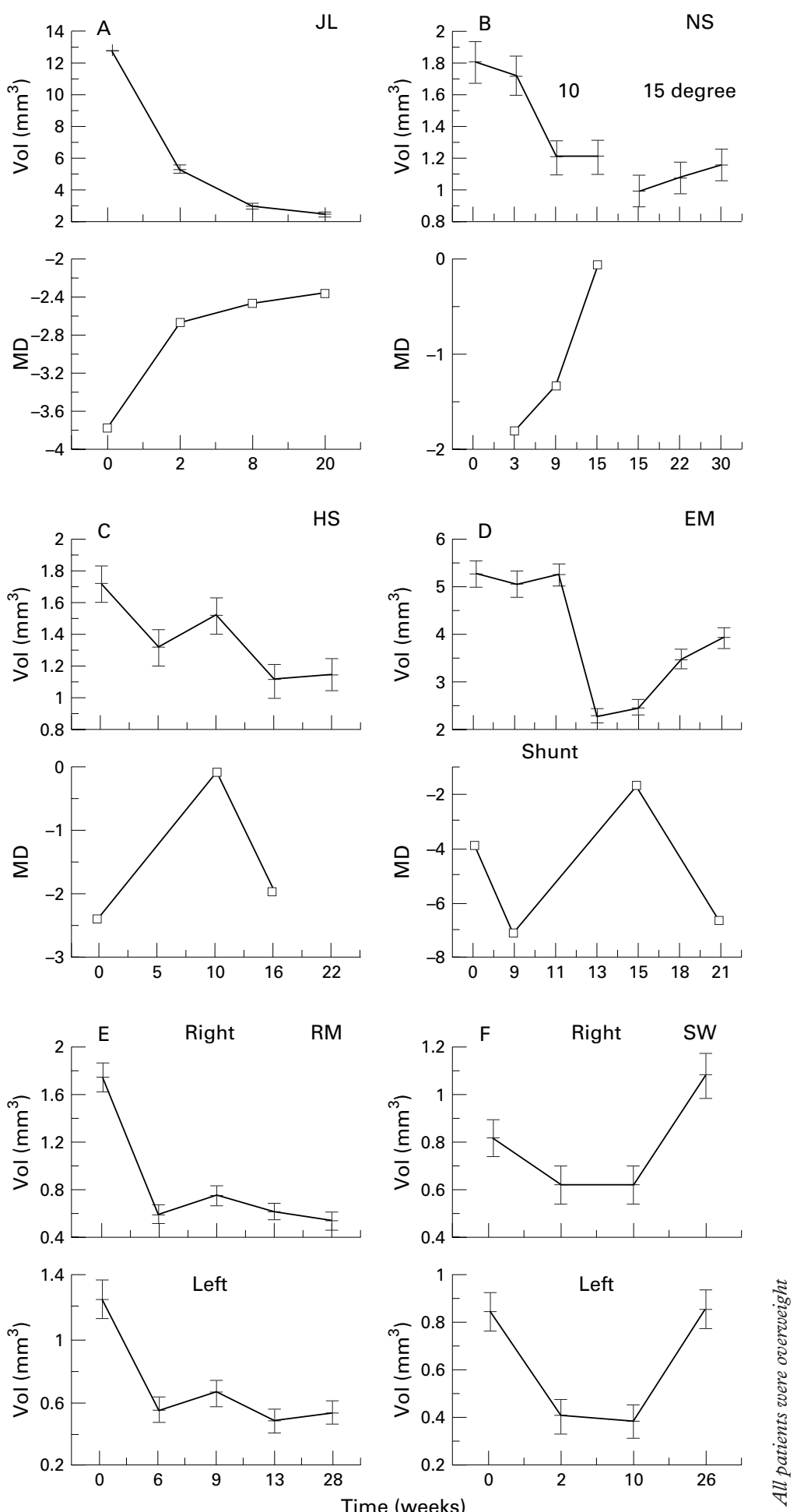

Figure 1 (A-D) Patients 1-4 showing one eye of each. All had symmetrical changes in disc swelling and field loss. Disc swelling (volume), upper graph. Field loss (mean deviation), lower graph. (A) Patient 1 . Marked disc swelling resolving to normal.

Resolution of field defect. (B) Patient 2. Resolution of mild disc swelling. Volume is then measured using a 15 degree field instead of 10 degree. Change in volume between the two lines is artefactual. Mild field loss shows improvement. (C) Patient 3. Resolution of mild disc swelling. Field loss improves initially with slight regression despite a normal disc. (D) Patient 4. Theco-peritoneal shunt was performed at 11 weeks with a rapid reduction in disc swelling and improvement in visual field. Subsequent worsening of both factors indicate shunt failure. (E) (F) Patients 5 and 6 had Goldmann fields only. (E) Patient 5.

Resolution of mild disc swelling symmetrically. (F) Patient 6. After the mild disc swelling resolves there is a significant late increase in volume symmetrically and asymptomatically. 

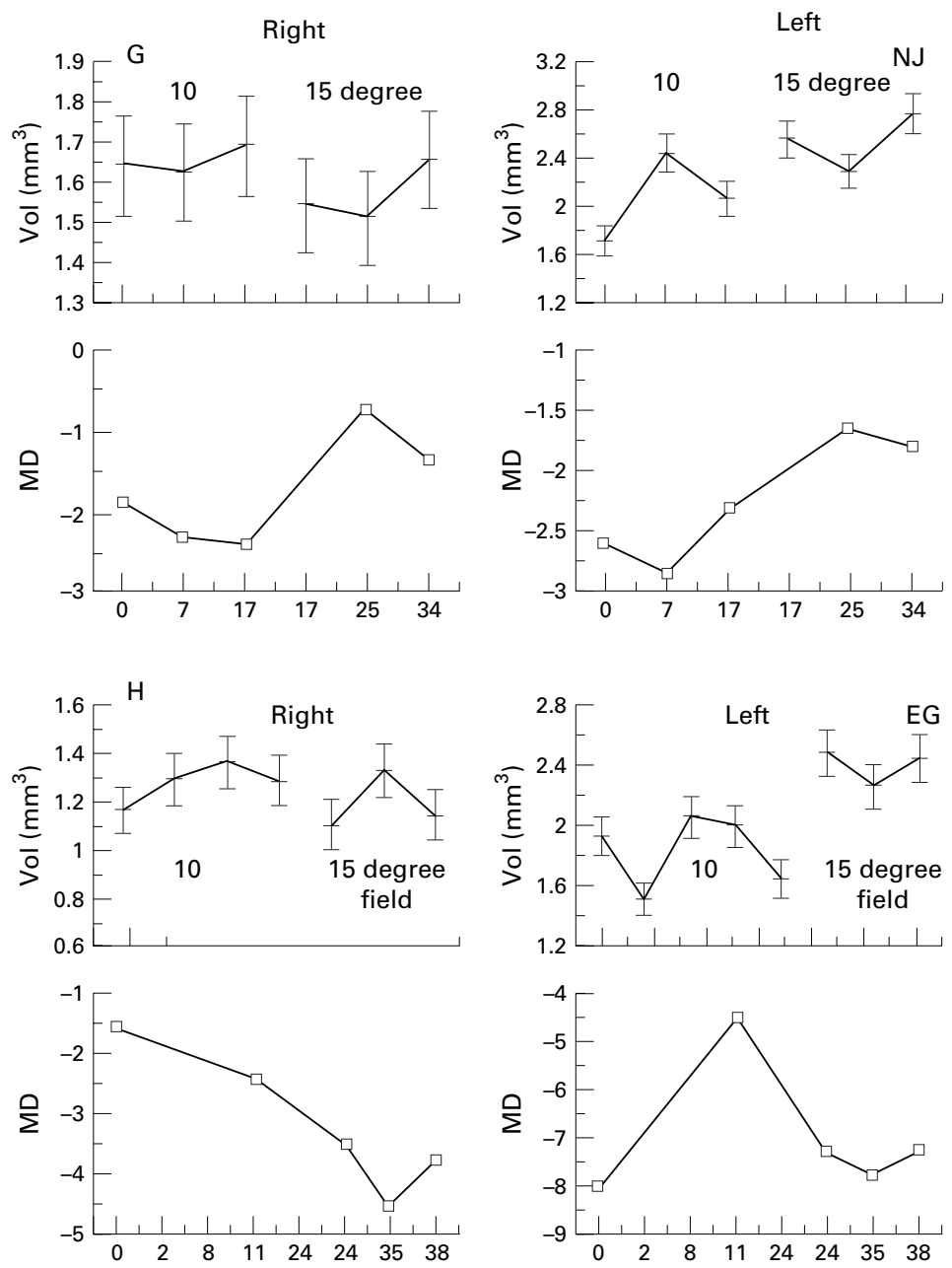

Time (weeks)

Figure 1 (continued) $(G, H)$ Patients 7 and 8. Fluctuating disc swelling with no overall significant change. Note the artefactual change in volume on changing to a 15 degree field. The left (more hypermetropic) disc is more swollen in both cases.

15 , or 20 degrees. A mean of three images is used to calculate disc volume. A circular contour line is placed around the swollen disc and a horizontal reference plane set at a depth of $50 \mu \mathrm{m}$ below a 4 degree segment on the temporal contour line. The volume of nerve head above the reference plane and within the contour line is generated (volume). SLO image acquisition time is approximately 1.5 seconds and each SLO examination takes only a few minutes.

Symmetry of swelling was compared at maximum volume in each patient using a contour line of equal diameter in each eye.

\section{Results}

To assess standard deviation (SD) and coefficient of variation of volume $(\mathrm{CoV})$ nine repeated images were obtained from each eye of all patients on one occasion. Repeated measurements from each patient showed the $\mathrm{SD}$ and $\mathrm{CoV}$ to depend on severity of disc swelling. The best estimate of SD is given by $0.048+0.0352 \times$ mean, where mean is of the nine single image disc volume measurements. $\mathrm{CoV}=100 \times \mathrm{SD} /$ mean. CoV3 (when a mean of three images is used to form volume) $=$
$\mathrm{CoV} / 3$. Therefore at volume $=1 \mathrm{~mm}^{3}, \mathrm{SD}=$ 0.08 and $\mathrm{CoV} 3=4.8 \%$. At volume $=5 \mathrm{~mm}^{3}$, $\mathrm{SD}=0.22$ and CoV3 $=2.6 \%$. Thus, $95 \%$ confidence intervals for the sequential measurements were calculated, as shown in Figure $1 \mathrm{~A}-\mathrm{H})$.

SYMMETRY OF DISC SWELLING

In all eight patients the clinical grade of papilloedema was symmetrical (Table 1). Volume measurements, however, showed significant differences between right and left eyes in all patients $(\mathrm{p}<0.05)$. This was only accompanied by asymmetrical symptoms and field loss in patients 7 and 8 who were also anisometropic.

Figure 1 demonstrates volume and mean deviation changes graphically. In patients 1,2 , 3 , and 4 (Fig 1A, B, C, D respectively) significant short term changes in volume were mirrored by corresponding changes in mean deviation. That is, increasing disc swelling is accompanied by worsening visual field and vice versa. Corrected pattern standard deviation (not shown) does not correlate well with mean deviation.

Patients 1-3, 5, and 6 followed a similar clinical course. After initiation of treatment disc volume decreased and stabilised at a normal level. Humphrey 30-2 fields in patients 1-3 showed initial mild, reproducible defects which resolved to normal. Early changes in disc swelling were not apparent ophthalmoscopically as the grade of papilloedema remained unchanged.

Patient 6 initially presented with normal Goldmann fields and severe headache which resolved. At last attendance volume has significantly increased again (to mild grade 1 papilloedema), without recurrence of symptoms (Fig 1F).

Patient 4 underwent theco-peritoneal shunting for deteriorating field and headache despite medical treatment. Two weeks post shunt there is a dramatic resolution of field loss and disc swelling. Over the following months both parameters deteriorated steadily. Increase in volume was apparent before recurrence of headache (Fig 1D).

Patients 7 and 8 show fluctuating field loss. Volume changes are often not significant and show no consistent relation to mean deviation change. In these cases a change in image field of view was made to ensure adequate size of contour line to encompass completely the swollen disc. Any change in volume during this is artefactual. The visual symptoms, field loss, and disc swelling in both are worse in the more hypermetropic eye (Table 1). Patient 7 complained of central visual distortion in the left eye only, while patient 8 complained of blurring and transient visual obscurations in the worse eye.

The wide spectrum of disc swelling varies from a volume of 1-2 $\mathrm{mm}^{3}$ - for example, patient 6 , requiring a 10 degree field of view (Fig 2) to a volume of $12-16 \mathrm{~mm}^{3}$ in patient 1 , requiring a 20 degree field of view (Fig 3). Both are shown at presentation and 2 weeks after commencement of treatment. 

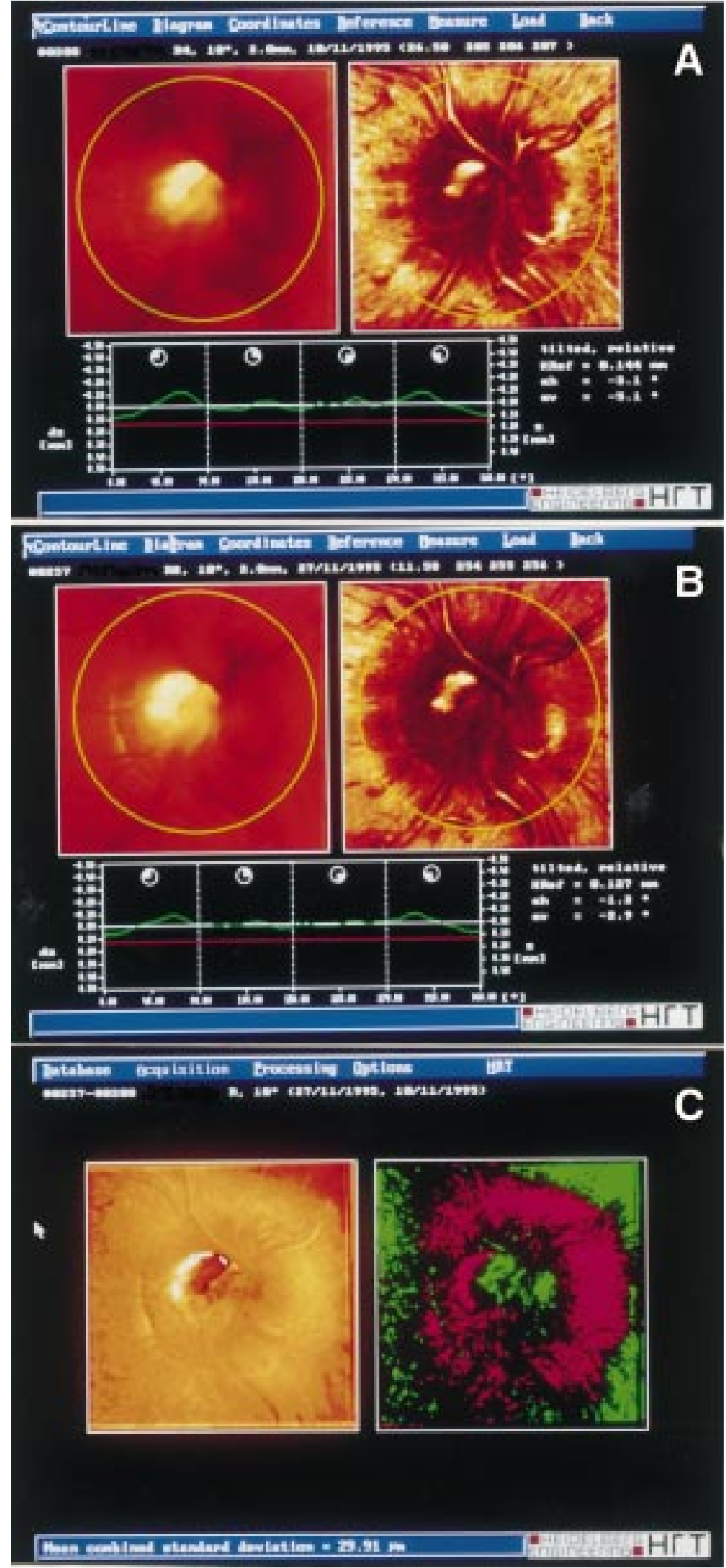

Figure 2 (A-C) Patient 6. Right eye. Mean topographic image (A) at presentation and (B) 2 weeks $\left(10^{\circ}\right.$ field of view). In the lower graph the height of the white line above the red represents mean height of the contour line above the reference plane. The green line represents height of contour line above reference plane in the clock hours shown. (C) Topographic difference image comparing the absolute height of each point between the images $(A)$ and $(B)$. Red represents decrease in disc swelling.
Patient 5 demonstrates that even small changes which can be difficult to appreciate clinically are easily detected.

\section{Discussion}

In the evaluation of optic nerve head swelling the primary goals are detection, characterisation, and monitoring. Funduscopy is clearly the best method to detect and characterise. Monitoring the degree of swelling funduscopically is subjective and small changes occur without a change in the clinical grade of papilloedema. This can be helped by stereophotography and retinal nerve fibre layer analysis. ${ }^{14}$ We evaluated the SLO, a relatively new method of quantifying papilloedema and show that it has potential uses in monitoring.

Visual loss in IIH most commonly occurs as a result of chronic papilloedema and secondary optic atrophy. It has been shown that visual loss is often reversible in IIH. ${ }^{1}$ Disc volume will decrease with successful therapeutic intervention. Volume may also decrease due to nerve fibre loss and secondary optic atrophy which would probably occur more slowly. So although the disc volume is helpful in the short term it must be interpreted in conjunction with symptomatology, funduscopy, and perimetry.

SLO may be particularly valuable in certain clinical situations. Firstly, once papilloedema has resolved and a baseline normal disc profile established, subtle but significant increases in swelling which may be asymptomatic can be detected. Secondly, it is a rapid, reliable indicator of response to therapeutic intervention. Reduction in volume in these cases is mirrored by improved symptoms and visual field. Perhaps it is more helpful in patients such as No 4 where a steady increase in disc volume confirms therapeutic failure and aids interpretation of field changes which can fluctuate in the short term. Thirdly, SLO is useful in a small subgroup of patients who have difficulty with automated perimetric techniques, giving an early indication of improvement or need for further treatment. Kinetic testing is not as sensitive and disc volume may demonstrate change sooner.

Quantification of swelling has demonstrated the wide range of disc swelling. From this small number it does not appear that the degree of disc swelling relates to visual loss. Duration of papilloedema is probably more important. The most significant and symptomatic visual loss was exhibited by patients 4,7 , and 8 , who all had only moderate but chronic ( $>6$ months) disc swelling.

From a technical perspective, there are several methods of setting the reference plane level. In all our images the horizontal plane above which volume is measured is set at 50 $\mu \mathrm{m}$ below a segment of temporal contour line. The contour line is of sufficient diameter to extend onto non-swollen peripapillary retina at this position. It is probable that with resolution of disc swelling a reduction in absolute height of the contour line above sclera in subsequent images does occur. But as this would lessen the measured volume reduction the significance of changes illustrated are, if anything, underesti- 

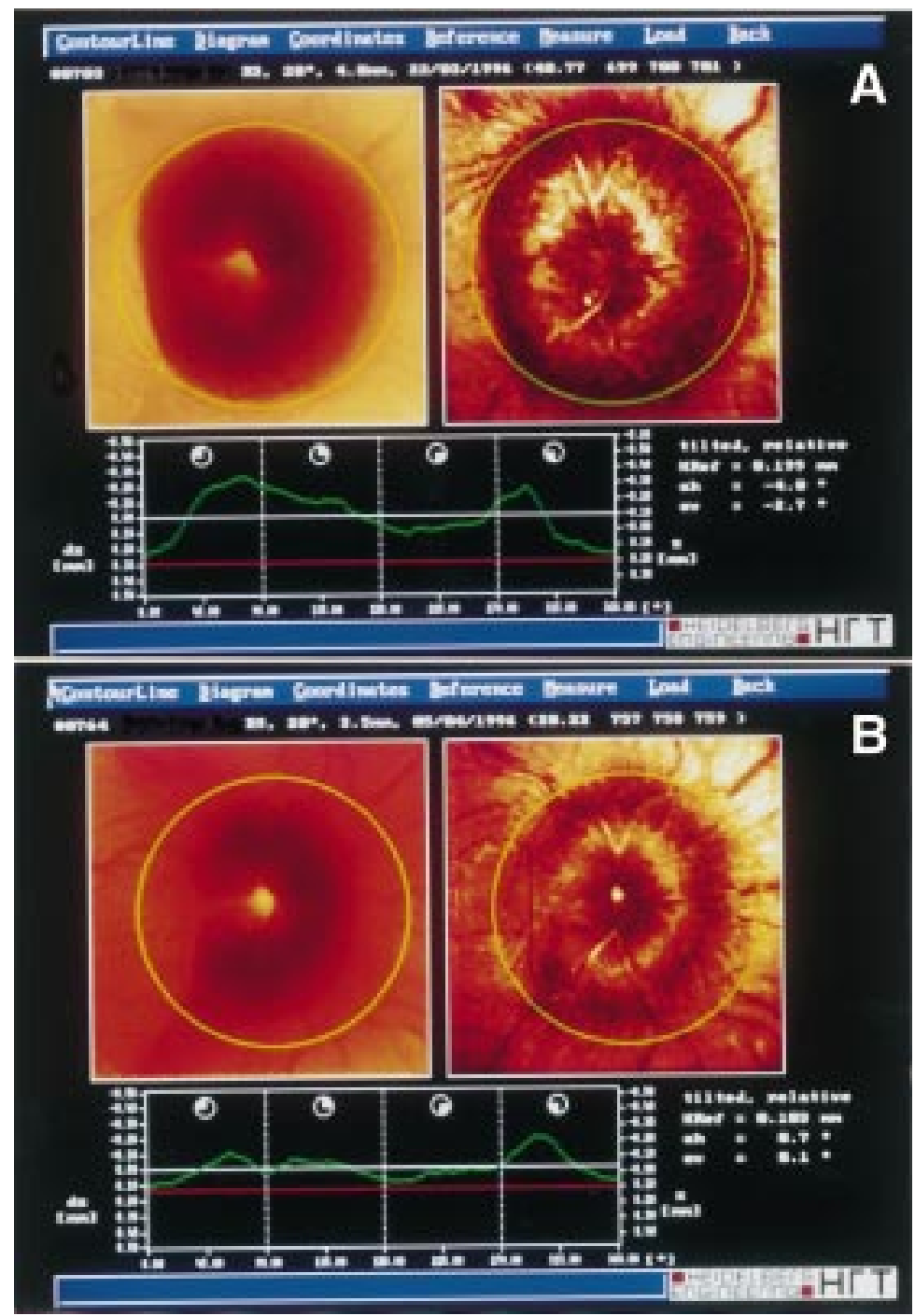

Figure 3 Patient 1. Right eye. (A) Presentation, (B) 2 weeks. Twenty degree field of view. Resolving, marked disc swelling. Note the change in contour line height.

mated. It is for this reason that in some patients the field of view was increased from 15 to 20 degrees to enable placement of a larger contour line in normal retina.

No single parameter produced by the SLO has been shown to be sufficiently sensitive to show short term changes in optic nerve head topography associated with glaucomatous progression. Changes in IIH are much larger and more rapid. When significant changes occur these are most often accompanied by a similar worsening or improvement of the visual field. The small changes in cases 7 and 8 are generally not significant, and correspondingly there are fluctuations but no definite trends in mean deviation. Therefore it seems that the SLO demonstrates changes which are likely to influence therapy.

We have deliberately analysed only the volume parameter although the images contain a wealth of information about disc topography which merits further study.

The scanning laser ophthalmoscope is an accurate tool for monitoring short term changes in optic nerve head swelling, making it a useful instrument in idiopathic intracranial hypertension. Visual fields remain the "gold standard" in the management but SLO provides additional valuable information.

1 Wall $\mathrm{M}$, George D. Idiopathic intracranial hypertension. A prospective study of 50 patients. Brain 1991;114:155-80. 2 Corbett JJ, Savino PJ, Thompson HS, et al.Visual loss in pseudotumor cerebri: follow up of 57 patients from 5 to 41 years and a profile of 14 patients with permanent severe visual loss. Arch Neurol 1982;39:461-74.

3 Corbett JJ, Thompson HS. The rational management of idiopathic intracranial hypertension. Arch Neurol 1989;46: 1049-51.

4 Wall M, George D. Visual loss in pseudotumour cerebri: incidence and defects related to visual field strategy. Arch Neurol 1987;44:170-5.

5 Digre KB, Corbett JJ. Pseudotumor cerebri in men. Arch Neurol 1988;45:866-72.

6 Orcutt JC, Page NGR, Sanders MD. Factors affecting visual loss in benign intracranial hypertension. Ophthalmology 1984;91:1303-12.

7 Smith TJ, Baker RS. Perimetric findings in pseudotumour cerebri using automated perimetric techniques. Ophthalmology 1986;93:887-94.

8 Corbett JJ, Nerad JA, Tse DT, et al. . Results of optic nerve sheath fenestration for pseudotumour cerebri: the lateral orbitotomy approach. Arch Ophthalmol 1988;106:1391-7.

9 Sanders MD. A classification of papilloedema based on a fluorescein angiographic study of 69 cases. Trans Ophthalmol Soc UK 1969;89:177-92.

10 Frisén L. Swelling of the optic nerve head: a staging scheme. f Neurol Neurosurg Psychiatry 1982;45:13-18.

11 Smith JL. Whence pseudotumour cerebri? $f$ Clin Neuroophthalmol 1985;5:55-6.

12 Weinreb RN, Lusky M, Bartsch DU, et al. Effect of repetitive imaging on topographic measurements of the optic nerve head. Arch Ophthalmol 1993;111:636-8.

13 Mikelberg FS, Wijsman K, Schulzer M. Reproducibility of topographic parameters obtained with the Heidelberg retina tomograph. F Glaucoma 1993;2:101-3.

14 Hedges TR III, Legge RH, Peli E, et al. Retinal nerve fibre layer changes and visual field loss in idiopathic intracranial hypertension. Ophthalmology 1995;102:1242-7. 